\title{
RESEARCH
}

Open Access

\section{Neurological phenotypes in patients with NLRP3-, MEFV-, and TNFRSF1A low- penetrance variants}

Elisabeth Mulazzani ${ }^{1,2}$, Danny Wagner ${ }^{1}$, Joachim Havla ${ }^{1}$, Miriam Schlüter ${ }^{1}$, Ingrid Meinl ${ }^{1}$, Lisa-Ann Gerdes ${ }^{1}$ and Tania Kümpfel ${ }^{*}$

\begin{abstract}
Background: Neurological manifestations and the co-occurrence of multiple sclerosis (MS) have been reported in patients with autoinflammatory diseases (AID) and variants of the NLRP3-, MEFV-, or TNFRSF1A gene. However, type and frequency of neurological involvement are widely undetermined.

Methods: We assessed clinical characteristics of 151 (108 with MS) patients carrying NLRP3-, MEFV- and TNFRSF1A low-penetrance variants from the Institute of Clinical Neuroimmunology. We evaluated demographic, genetic, and clinical features with a focus on central nervous system (CNS) involvement including magnetic resonance imaging (MRI) results and cerebrospinal fluid (CSF) data. The disease course of AID patients with MS was compared to a matched MS control group without mutations.

Results: The genetic distribution comprised 36 patients (23\%) with NLRP3- and 66 patients (43\%) with TNFRSF1A low-penetrance variants as well as 53 (34\%) patients carrying pathogenic mutations or low-penetrance variants in the MEFV gene. MS patients displayed most frequently the R92Q TNFRSF1A variant $(n=51 ; 46 \%)$ followed by the Q703K NLRP3 variant $(n=15 ; 14 \%)$ and the E148Q substitution $(n=9 ; 8 \%)$ in the MEFV gene. The disease course of MS was not influenced by the genetic variants and did not differ from MS patients $(n=51)$ without mutations. AID patients without MS most frequently harbored MEFV mutations ( $n=19,43 \%)$ followed by NLRP3- $(n=17,39 \%)$ and TNFRSF1A ( $n=8,18 \%$ ) low-penetrance variants. Sixteen (36\%) of them suffered from severe CNS involvement predominantly recurrent aseptic meningoencephalitis and optic neuritis accompanied by abnormal MRI and CSF results. Severe CNS inflammation was associated with the Q703K allele. Headache was a highly prevalent neurological symptom (up to 74\%), irrespective of the underlying genetic variation. The NLRP3 cohort without MS more frequently exhibited affections of the cranial nerves $(C N)(p=0.0228)$ and motor symptoms $(p=0.0455)$. Elevated acute-phase reactants were detected in all patients, and fever episodes were present in up to 50\%. Arthralgias were the most frequently identified constitutional symptom among all subgroups.

(Continued on next page)
\end{abstract}

\footnotetext{
* Correspondence: tania.kuempfel@med.uni-muenchen.de

'Institute of Clinical Neuroimmunology, Biomedical Center and University

Hospital, Ludwig-Maximilians University, Marchioninistraße 15, 81377 Munich, Germany

Full list of author information is available at the end of the article
}

(C) The Author(s). 2020 Open Access This article is licensed under a Creative Commons Attribution 4.0 International License, which permits use, sharing, adaptation, distribution and reproduction in any medium or format, as long as you give appropriate credit to the original author(s) and the source, provide a link to the Creative Commons licence, and indicate if changes were made. The images or other third party material in this article are included in the article's Creative Commons licence, unless indicated otherwise in a credit line to the material. If material is not included in the article's Creative Commons licence and your intended use is not permitted by statutory regulation or exceeds the permitted use, you will need to obtain permission directly from the copyright holder. To view a copy of this licence, visit http://creativecommons.org/licenses/by/4.0/ The Creative Commons Public Domain Dedication waiver (http://creativecommons.org/publicdomain/zero/1.0/) applies to the data made available in this article, unless otherwise stated in a credit line to the data. 
(Continued from previous page)

Conclusions: Our data highlight the high prevalence of neurological manifestations, including concomitant MS, among NLRP3-, MEFV-, and TNFRSF1A low-penetrance variants. In particular, patients carrying the Q703K NLRP3 variant are at risk for severe CNS inflammation and CN affection.

Keywords: CAPS, TRAPS, FMF, Multiple sclerosis, Autoinflammation, Autoimmunity

\section{Background}

Cryopyrin-associated periodic syndromes (CAPS) including Muckle-Wells syndrome (MWS), familial cold autoinflammatory syndrome (FCAS) and neonatal-onset multisystem inflammatory disease (NOMID/CINCA), familial Mediterranean fever (FMF), and tumor necrosis factor receptor-associated periodic syndrome (TRAPS), all belong to a group of rare, monogenetic autoinflammatory diseases (AID) caused by a dysregulation of the innate immune system. While CAPS is mediated via gain-of-function mutations in the NLRP3 gene leading to a constitutively activated NLRP3 inflammasome, FMF is caused by sequence variants in the $M E F V$ locus encoding the inflammasome complex pyrin $[1,2]$. At the molecular level, NLRP3 serves as an intracellular danger signaling complex, while pyrin acts as a sensor detecting an imbalance in RhoGTPases activity [3]. Despite this diverging signaling pathway upstream of inflammasome activation exists, both CAPS and FMF result in a common overproduction of interleukin $1 \beta(\mathrm{IL}-1 \beta)[4,5]$. On the contrary, TRAPS is caused by mutations in the TNFRSF1A gene, which encodes the tumor necrosis factor receptor (TNF) receptor, and has formerly been associated with an accumulation of mutated TNFR1 receptors in the endoplasmic reticulum due to inadequate shedding, thus triggering inflammatory response [6-8].

Clinically, AID are characterized by unexplained episodes of fever and systemic inflammation involving joints, skin, muscles, eyes, and serosal surfaces accompanied by elevated acute-phase reactants [9]. Neurological manifestations with the involvement of the central nervous system (CNS) including aseptic meningitis, headache, increased intracranial pressure, seizures, cerebral vasculitis, and inflammatory lesions in the brain have mainly been reported in CAPS, but are also present in FMF and TRAPS and may even dominate the clinical picture [10-18]. Furthermore, cases with multiple sclerosis (MS)-like presentations have been observed for all three of them [17, 19-21]. The importance of variants with unknown pathogenic significance (such as p.R92Q in TNFRSF1A or p.Q703K in NLRP3) is still under debate, and their clinical phenotypes are often challenging for clinicians [22, 23]. In recent studies, we could describe several patients with
NLRP3-, MEFV-, and TNFRSF1A low-penetrance variants in association with inflammatory diseases of the CNS including MS [24-29].

The aim of this study was to evaluate genotypephenotype correlations focusing on neurological manifestations in a monocentric cohort of 151 patients (with and without (w/o) MS) with predominantly lowpenetrance mutations in the NLRP3-, MEFV-, or TNFRSF1 gene.

\section{Methods}

All patients were consecutively seen and examined by an experienced neurologist at our neuroimmunological outpatient clinic from 2006 to 2020, which is specialized on the diagnosis and treatment of adult patients with MS and other neuroimmunological diseases. Inclusion criteria encompassed clinical presentation suggestive of AID ( $\geq 2$ symptoms compatible with AID) and the genetic proof of a variant in the NLRP3-, MEFV-, or TNFRSF1A genes. Genetic testing included sequencing of exons 3,4 , and 6 of the NLRP3 gene as well as exons 2, 3, and 10 of the $M E F V$ gene and exons 2, 3, 4, and 6 of the TNFRSF1A gene. Patients were then grouped according to their AID and MS status into NLRP3-, MEFV-, and TNFRSF1A variants with and w/o MS. Patients harboring genetic mutations in two different genes were accordingly allocated to both subgroups. In addition, MS disease course of mutation carriers was compared to a matched MS control group $(n=51)$ lacking genetic variants in the NLRP3-, MEFV-, and TNFRSF1A genes.

Demographic data, family history, clinical characteristics, MRI, and laboratory findings (cerebrospinal fluid [CSF] and blood), as well as treatment information, were collected during standardized visits at our institute. In patients with MS, data on the disease course were evaluated using the Expanded Disability Status Scale (EDSS) and the Multiple Sclerosis Severity Scale (MSSS) was evaluated [30]. MRI and CSF data were analyzed in more detail in AID patients w/o MS, and CNS involvement was classified as mild (only clinical signs/symptoms, normal CSF, no MRI abnormalities) or severe (clinical signs/symptoms, abnormal CSF and/or MRI). In addition, neurological symptoms were specifically grouped into 5 categories: sensory and motor symptoms, cerebellar signs, cranial nerve $(\mathrm{CN})$ affection, and headache. The term $\mathrm{CN}$ 
affection itself was defined as specific inflammation of the cranial nerves I-XII in patients with AID and CNS manifestation w/o MS. Clinical characteristics of constitutional symptoms were also assessed in all patients including disease course (chronic vs. episodic), fever, skin involvement, abdominal pain, arthralgias, myalgias, and ocular involvement (conjunctivitis/uveitis/retinitis/papillitis). All patients were classified according to the most recent classification criteria for AID [23]. Patients were followed over time whenever possible. Follow-up time of AID patients with MS (mean $10.5 \pm 4.6$ ) and of MS control group (mean 9.2 \pm 2.8 ) ranged from 2 to 18 years. Mean follow-up time of AID patients w/o MS was $8.7 \pm 3.1(4-15)$ and exact follow-up time for each patient is shown in suppl. fig. 1 .

Frequencies and percentages were used as descriptive statistics for categorical variables. Median and range were used to describe numerical variables. In order to analyze the clinical phenotype of the patients in relation to their gene variants and MS disease status, we divided the patients into six subgroups: NLRP3 variants without (w/o) or with MS, MEFV variants w/o or with MS, TNFRSF1A w/o or with MS. Intergroup comparisons of main clinical characteristics were assessed by Fisher's exact test or chi ${ }^{2}$ test, as appropriate. All tests were twosided. All analyses were performed with Prism Software (GraphPad@). The Benjamini-Hochberg procedure was used to correct for multiple comparisons, and the threshold for statistical significance was set to $p<0.05$.

\section{Results}

\section{Demographic and general clinical characteristics}

Altogether, 151 patients (40 males, 111 females mean age 44 , range $20-75$ years) with low-penetrance variants or pathogenic mutations in the NLRP3-, MEFV-, or TNFRSF1A gene and clinical presentation suggestive for AID were included in the study. One hundred and eight patients had a concomitant diagnosis of MS. Four of 151 patients carried a double mutation (1 patient w/o MS: NLRP3 and TNFRSF1A variants, 3 patients with MS: two with MEFV and TNFRSF1A variants, one with MEFV and NLRP3 variants) leading to 155 AID in total (AID w/o MS = 44; AID with MS = 111) (Table 1). The majority of patients were adults with a median age at AIDrelated symptom onset of $27.5 \pm 6.8$ years consistent with late-onset disease manifestation. None of the patients were diagnosed before the age of 6 months. The longest diagnostic latency for AID was observed in MS patients with MEFV variants with a mean of $12.3 \pm 11.4$ years. Only $38(25 \%)$ patients were of Mediterranean origin, most of them carried MEFV variants $(n=22)$. Overall family history for AID was positive in 68 (44\%) patients, with more affected family members in patients with NLRP3 variants w/o MS (65\%, see Table 1). A total of $17(11 \%)$ patients (4 with MS) were treated with anti-
IL1 drugs (anakinra or canakinumab), while 20 (13\%) patients (all with MEFV variants, 4 with MS) received colchicine. Detailed characteristics of all patient groups are presented in Table 1.

\section{Genetic data}

In total, 4 different variants were found in the NLRP3 gene, 15 mutations were identified in the $M E F V$ gene, and 6 genetic variants were found in the TNFRSF1A gene. The vast majority of patients carried lowpenetrance mutations and was heterozygous for the respective mutation.

In detail, 36 patients (23\%) showed a low-penetrance variant in the NLRP3 gene $(78 \%$ carried a Q703K variant in exon 3), 53 patients (34\%) exhibited pathogenic mutations or low-penetrance variants in the $M E F V$ gene $(26 \%$ with the E148Q variant in exon 2) and 66 patients (43\%) showed variants in the TNFRSF1A gene $(85 \%$ with the R92Q variant in exon 4). A complete overview of all genetic variants is given in Fig. 1a-c. In 13 (25\%) FMF patients ( $10 \mathrm{w} / \mathrm{o}$ MS, three with MS), a compound mutation was identified. Severe disease-causing FMF mutations including M694V (+/-), M694V (+/+), K695R $(+/-)$, and M680I $(+/-)$ were found in $22(42 \%)$ MEFV mutation carriers.

AID patients w/o MS $(n=40)$ most frequently harbored MEFV mutations $(n=19,43 \%)$ followed by NLRP3- $(n=17,39 \%)$ and TNFRSF1A $(n=8,18 \%)$ lowpenetrance variants (Fig. 1). AID patients with MS $(n=$ $111)$ displayed most frequently the $R 92 \mathrm{Q}$ variant $(n=$ 51; 46\%) in the TNFRSF1A gene followed by the Q703K variant in the NLRP3- $(n=15 ; 14 \%)$ and the E148Q variant $(n=9 ; 8 \%)$ in the MEFV gene (Fig. 1). The MEFV low-penetrance variants K695R $(n=7)$ and R408Q $(n=$ 8 , in six cases as a compound heterozygous P369SR408Q variant) were almost exclusively found in the MS cohort (Fig. 1b).

\section{Neurological manifestation Patients without MS}

Taken together, 95\% of AID patients w/o MS showed neurological manifestations, 16 (36\%) of those suffered from severe CNS involvement. Genetically, significantly more patients with severe CNS involvement harbored a Q703K variant in the NLRP3 gene $(n=11 ; 69 \%)$ compared to MEFV- $(n=3,19 \%)$ and TNFRSF1A $(n=2$, 13\%) low-penetrance variants ( $\mathrm{chi}^{2}$ test: $\left.p=0.0228\right)$. The clinical picture of severe CNS involvement was dominated by optic neuritis $(n=11 ; 69 \%)$ and aseptic meningitis/meningoencephalitis $(n=5 ; 31 \%)$ while one patient suffered from cerebral vasculitis $(n=1 ; 3 \%)$.

Headache syndromes were frequently observed among all AID subgroups w/o MS and most commonly found in patients with MEFV- $(n=14 ; 74 \%)$ followed by 


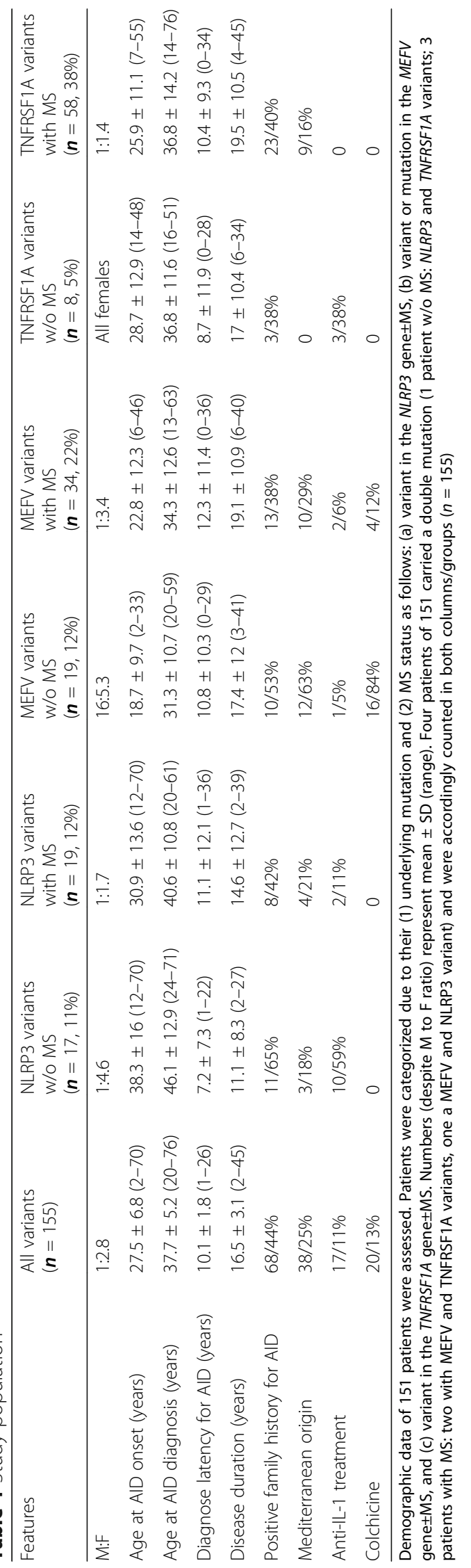




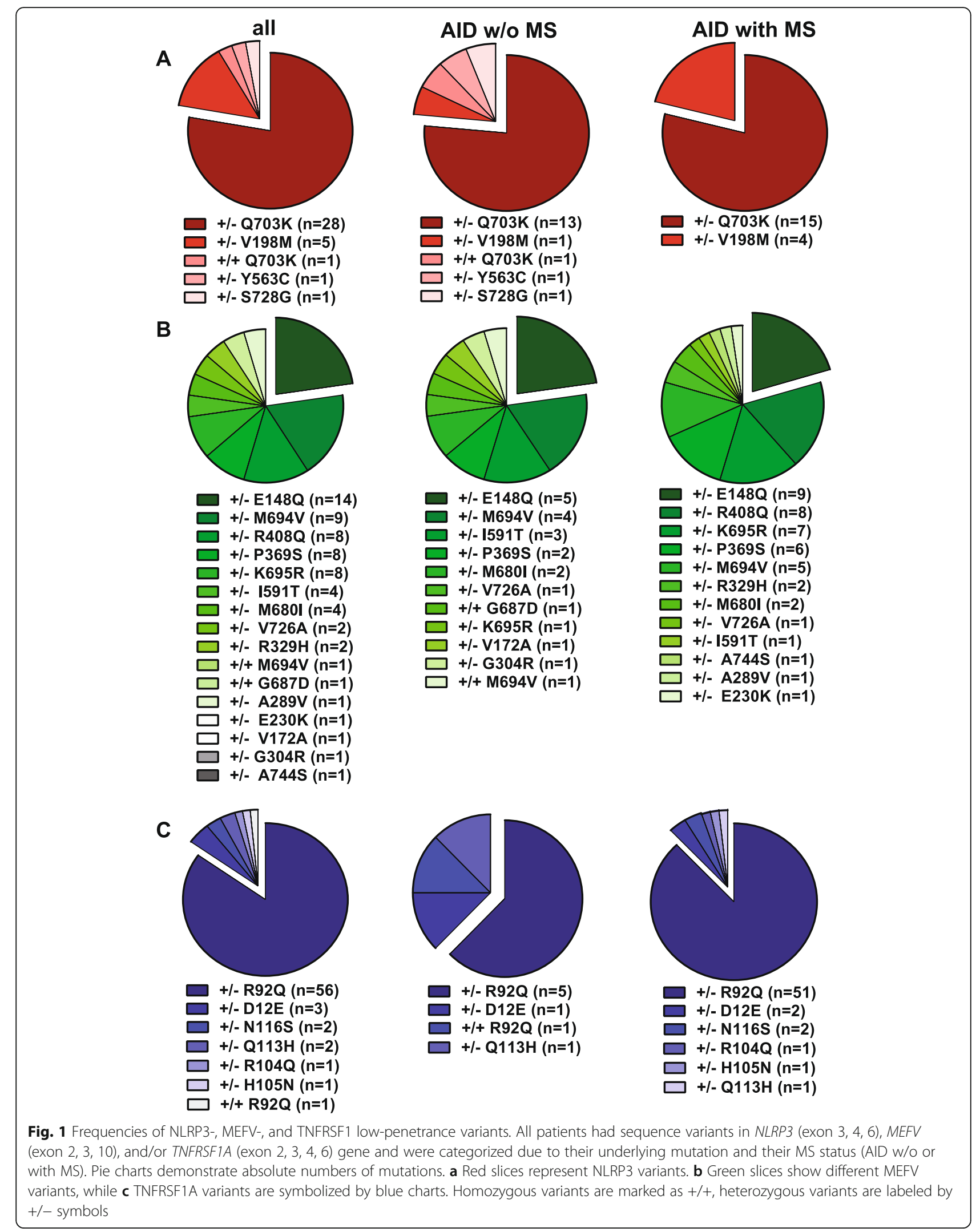



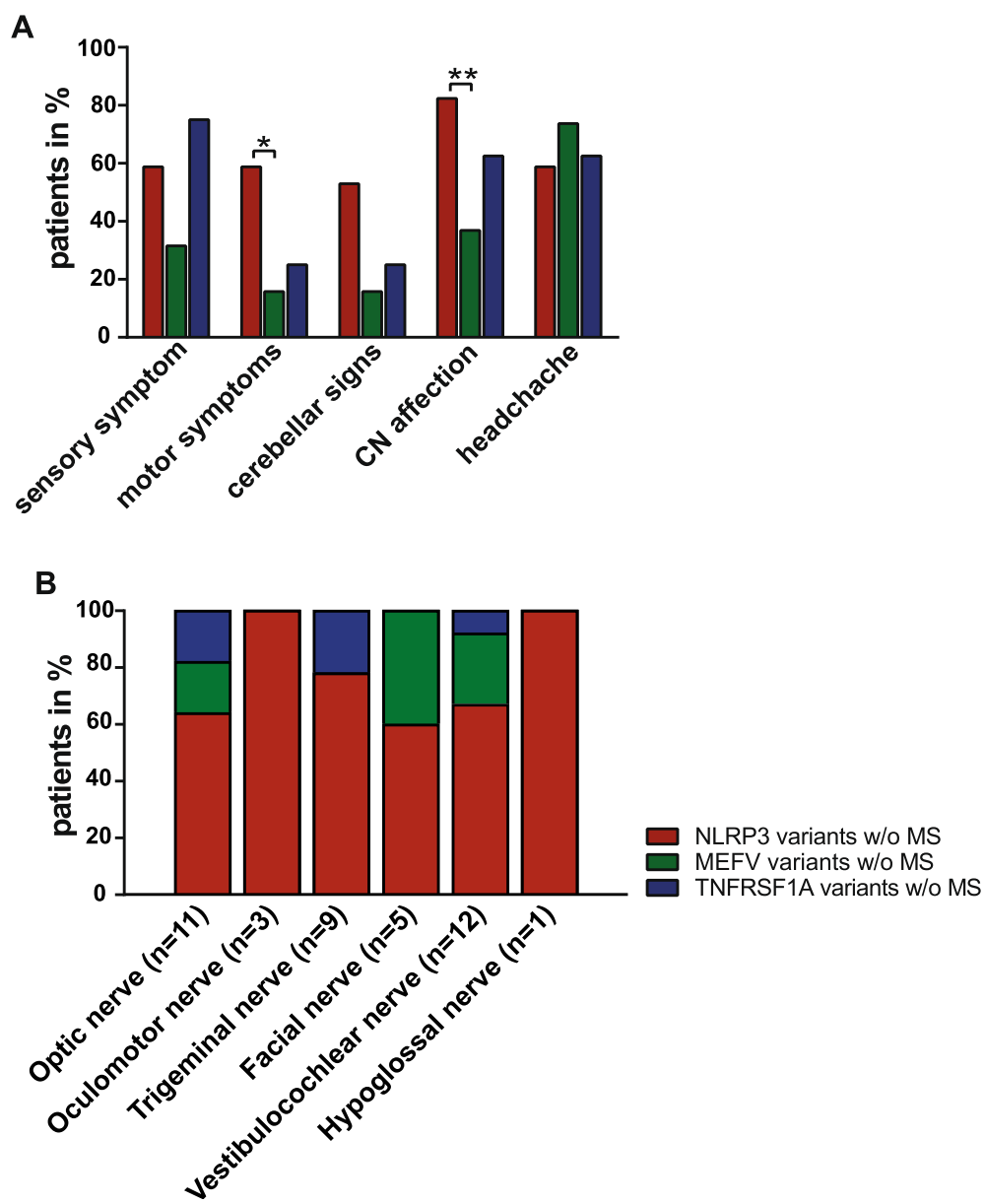

Fig. 2 Neurological symptoms of AID patients w/O MS. Neurological symptoms were distinguished as follows: a sensory and motor symptoms, cerebellar signs, cranial nerve (CN) affection and headache syndromes in NLRP3-, MEFV-, and TNFRSF1A low-penetrance variants w/o MS. b Distribution of patients W/o MS and NLRP3-, MEFV-, and TNFRSF1A low-penetrance variants and CN affection are shown as percentages

TNFRSF1A- $(n=5 ; 63 \%)$ and NLRP3 low-penetrance variants $(n=10,59 \%)$. Patients with NLRP3 variants were also more commonly affected by motor symptoms (NLRP3 variants: $n=10,59 \%$; vs. MEFV variants: $n=3$, $16 \% ; p=0.0455)$ compared to MEFV low-penetrance variants (Fig. 2a). In NLRP3 low variant carriers, CN affection was significantly more often identified compared to MEFV patients (NLRP3 variants: $n=14,82 \%$; vs. MEFV variants: $n=7,37 \% ; p=0.0228)$. Distribution of NLRP3-, MEFV-, and TNFRSF1A low-penetrance variant carriers regarding $\mathrm{CN}$ affection are shown in Fig. $2 \mathrm{~b}$.

MRI data were available for 33 (75\%) AID patients w/o MS (NLRP3 variants: $n=16$; 94\%, MEFV variants: $n=10$; $53 \%$, and TNFRSF1A variants: $n=7 ; 88 \%)$. Abnormal MRI findings were present in 11 (69\%) patients with NLRP3 low-penetrance variants, in six (60\%) patients with MEFV variants, and in three (43\%) patients with TNFRSF1A variants. Unspecific white matter lesions were the most common neuroradiological feature. A disrupted blood-brain barrier indicated by an increased gadolinium uptake was only seen in patients with the Q703K NLRP3 variant. Further morphological MRI features are listed in Fig. 3.

CSF data were available in 31 (71\%) AID patients w/o MS (NLRP3 variants: $n=15 ; 88 \%$, MEFV variants: $n=$ 10; $53 \%$, and TNFRSF1A variants: $n=6$; 75\%). Pathological CSF findings were present in ten of NLRP3(67\%), two of MEFV- (20\%), and two of TNFRSF1A (33\%) low-penetrance variants (Fig. 4).

\section{MS patients}

A total of 108 patients with AID had a concomitant MS. The majority of patients $(n=92)$ were classified as relapsing-remitting MS (RRMS), twelve had secondary progressive MS (SPMS), and seven patients were diagnosed with primary progressive MS (PPMS). Neurological presentations including headache, as well as MSrelated disease course, disability, and severity, scores did not differ among AID groups (Fig. 5a, b) and were comparable to MS patients without mutations (Table 2). MS-related medications consisted of disease-modifying 

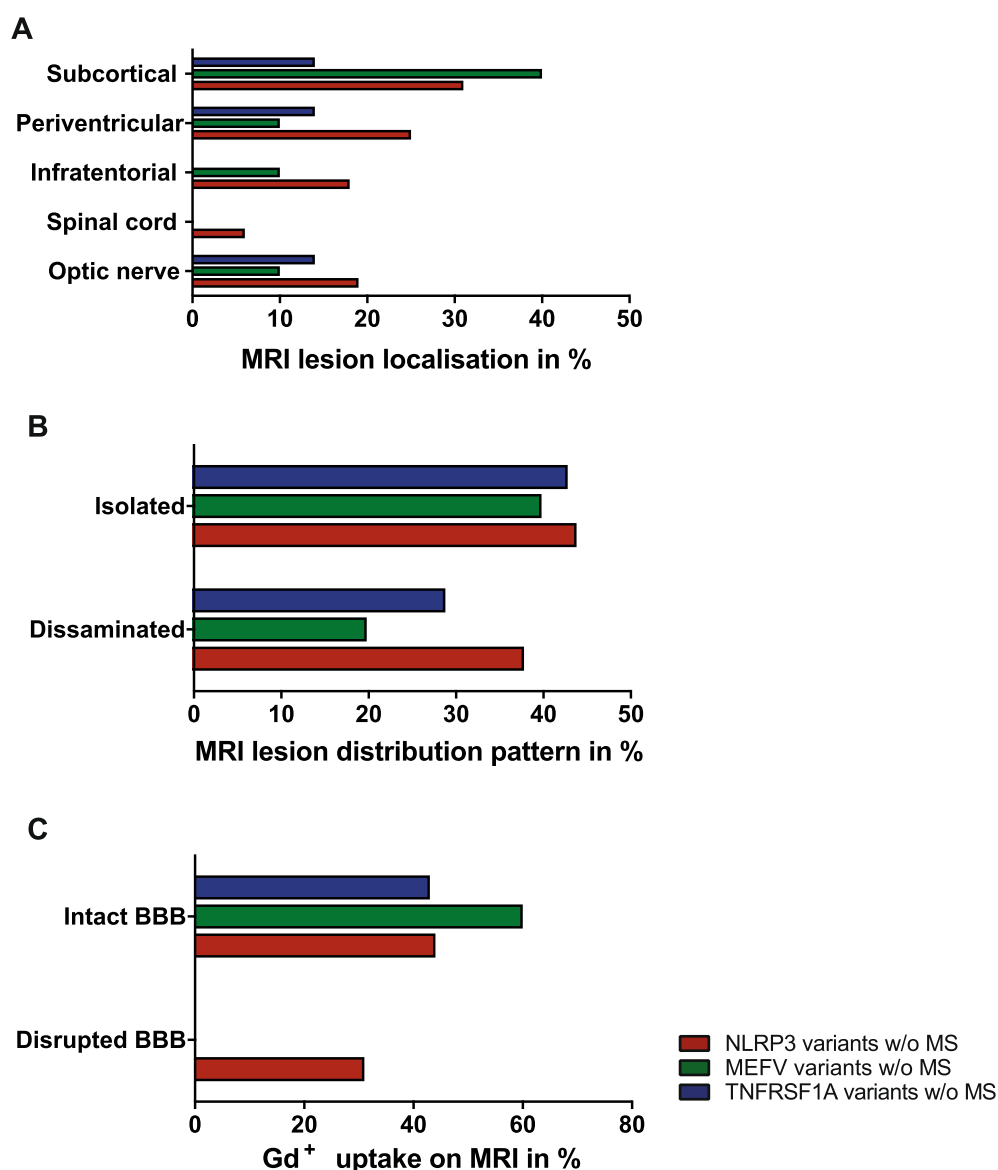

Fig. $3 \mathrm{MRI}$ data of AID patients w/o MS. MRI data of low-penetrance mutation carriers in the NLRP3-, MEFV-, or TNFRSF1A gene w/o MS are shown as percentages

therapies (DMT) for mild disease course in $67 \mathrm{MS}$ patients $(60 \%)$ and for active disease course in $31 \mathrm{MS}$ patients (28\%). A slightly higher proportion of MS patients with AID was more often treated with DMT for active disease compared to the MS control group and also showed more often a positive family history for MS. Detailed clinical features of MS patients with AID and of MS control group are summarized in Table 2.

\section{Systemic symptoms}

Overall $79 \%(n=119)$ of patients fulfilled the most recent classification criteria for the respective AID [23]: $58 \%(n=$ $21)$ with NLRP3 variants, $60 \%(n=32)$ with MEFV lowpenetrance or pathogenic mutations, and $100 \%(n=66)$ with TNFRSF1A variants. Most patients suffered from an episodic disease course $(n=137 ; 88 \%)$ with intermittent flares. Looking at all patients, arthralgias was the most common symptom followed by abdominal pain, myalgias, skin rash, fever and ocular involvement (Fig. 6a). Elevated acute-phase reactants (CRP $2.7 \pm 1.6$ [range 0.6-86] and SAA $31 \pm 36.2$ [range 5.2-3115]) were detected in all subgroups (Table 3). None of the patients so far showed evidence of amyloidosis or kidney disease. Twenty-two patients $(14 \%)$ were also diagnosed with other autoimmune diseases including autoimmune thyroid disease, diabetes mellitus type I, Crohn's disease and alopecia areata. Four patients had a concomitant diagnosis of a rheumatic disease (two patients with D12E and N116S variants in the TNFRSF1A gene had rheumatoid arthritis/polyarthritis, one patient with a M694V MEFV variant was diagnosed with systemic lupus erythematosus, and one patient with a compound P369/R408Q MEFV variant was diagnosed with ankylosing spondylitis).

\section{Patients without MS}

Patients w/o MS and with TNFRSF1A variants showed significant more often myalgias $(n=7 ; 88 \%)$ compared to patients with MEFV variants $(n=7 ; 37 \%, p=0.0169)$. Patients with TNFRSF1A variants $(n=8 ; 100 \%)$ also showed significant more often skin rashes compared to patients with MEFV variants $(n=4 ; 21 \%, p=0.0074)$ (Fig. 6b). 


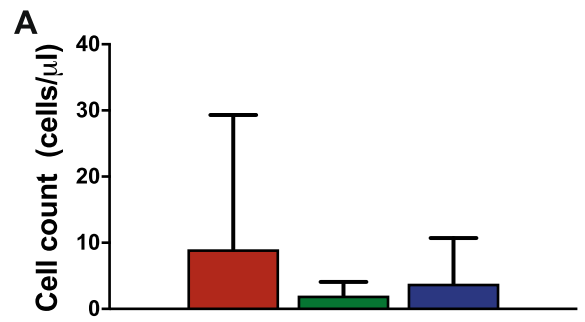

B

C
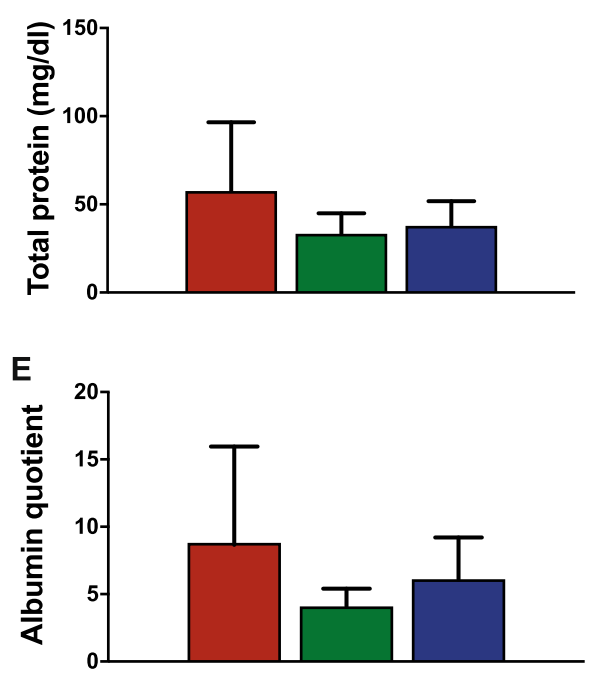

G

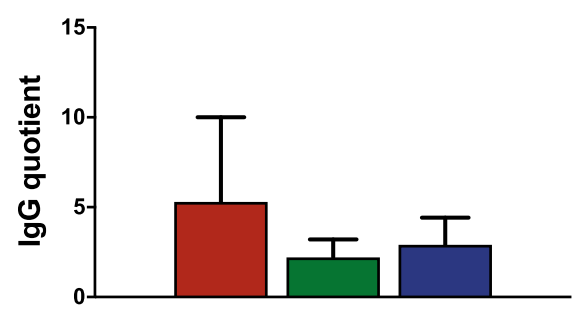

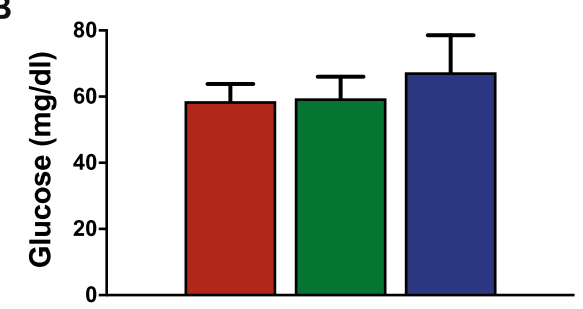

D

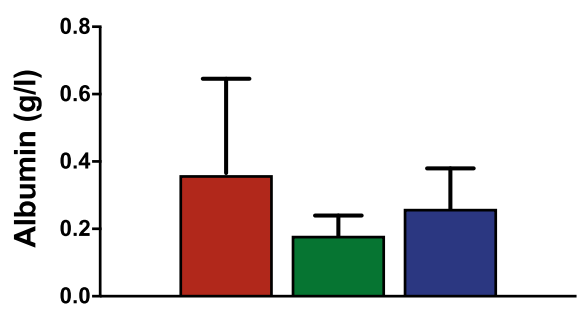

F

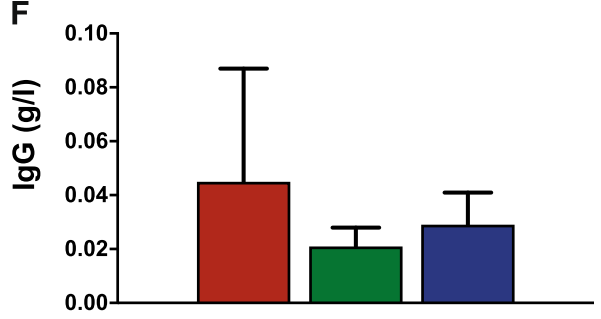

H

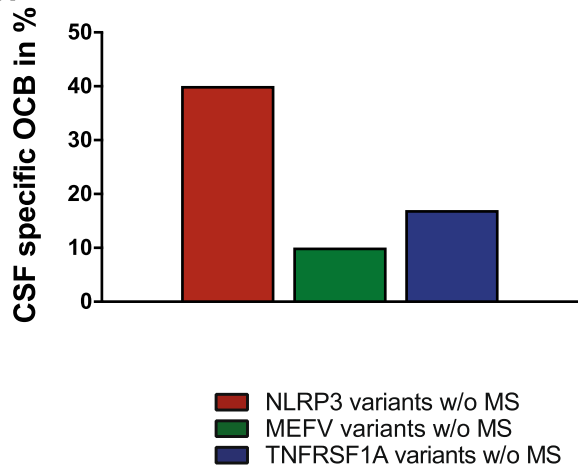

Fig. 4 CSF data of AID patients w/o MS. CSF data of low-penetrance mutation carriers in the NLRP3-, MEFV-, or TNFRSF1A gene w/o MS are shown as mean \pm SD or as percentages (OCB)

\section{Patients with MS}

MS patients with NLRP3 low-penetrance variants $(n=4$; $21 \%)$ suffered less frequently from gastrointestinal symptoms compared to MS patients with TNFRSF1A variants ( $n=36 ; 62 \%, p=0.0169)$. Ocular involvement specifically uveitis was most commonly identified in NLRP3 low-penetrance variants $(n=12 ; 63 \%)$ in comparison to TNFRSF1A- $(n=14 ; 24 \%, p=0.0169)$ and MEFV variants $(n=6 ; 18 \%, p=0.0141)$ (Fig. 6c). Neither treatment with colchicine nor with anti-IL-1 therapy (Table 1 ) had a negative impact on the disease course of MS.

\section{Discussion}

This large, monocentric case series demonstrates a broad spectrum and high prevalence of neurological presentations in adult patients with AID predominantly caused by a variety of low-penetrance mutations in the NLRP3-, $M E F V^{-}$, or TNFRSF1A gene. Here, $95 \%$ of AID patients w/o MS suffered from neurological manifestations. This highlights the importance of neurological assessments in patients with AID and conversely, that neurologists may consider AID in patients with unexplained CNS as well as systemic symptoms caused by inflammation. 

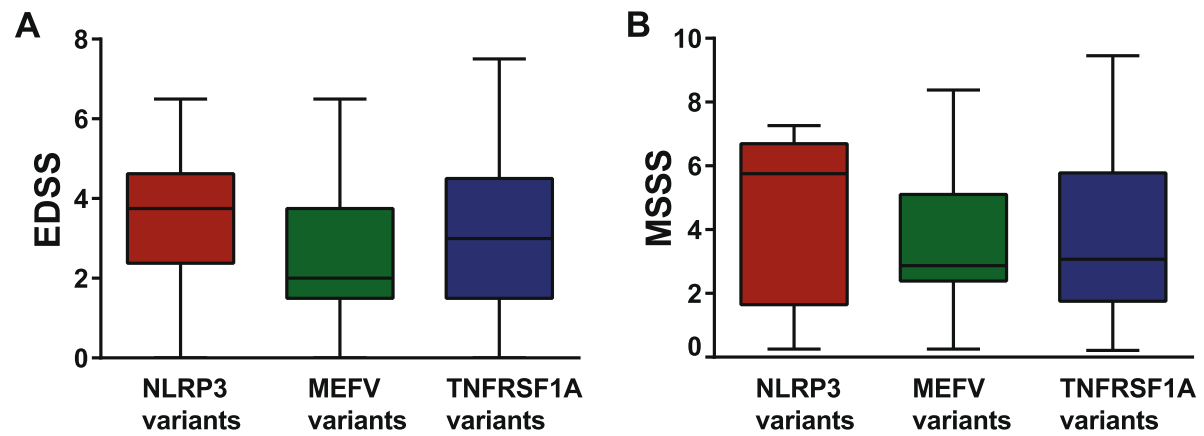

Fig. 5 Disability and severity scores of AID patients with MS. Expanded Disability Status Scale (EDSS) and the Multiple Sclerosis Severity Scale (MSSS) in NLRP3-, MEFV-, and TNFRSF1A low-penetrance variants with MS are shown at initial presentation

Low-penetrance variants occur at a high allelic frequency in the common population [26, 28, 31, 32]. Clinically, they usually present with a "late-onset" of AID and a more heterogeneous spectrum of symptoms, often accompanied by a milder disease course compared to classical pathogenic mutations in patients with AID [3336]. Our results also show that the majority of patients had late onset of AID and a broad phenotype overlapping among the different subgroups. Nevertheless, one third of our patients had a severe phenotype involving the CNS. There is mounting evidence, based on both experimental and clinical studies, that also nonconfirmatory genotypes can exert pro-inflammatory effects and may lead to severe organ involvement including CNS manifestations in a subset of those patients [24,
37, 38]. We observed severe CNS manifestations including recurrent aseptic meningitis/meningoencephalitis and optic neuritis as well as cerebral vasculitis in several patients w/o MS, most of them harboring the Q703K NLRP3 variant. This was underscored by a high number of abnormal MRI and CSF findings in those patients. CNS inflammation is often observed in patients with pathogenic NLRP3 mutations but has also been reported in patients with MEFV mutations [5, 12, 14, 15, 39, 40]. Regarding NLRP3, neurological involvement was so far reported for $40-95 \%$ of CAPS patients and has mainly been linked to the pathogenic R260W mutation of the NLRP3 gene, while the V198M variant was negatively associated with neurological involvement $[12,13,35]$. Thus, the high occurrence of severe CNS inflammation in patients with

Table 2 Clinical data of NLRP3-, MEFV-, and TNFRSF1A low-penetrance variants with MS

\begin{tabular}{llllll}
\hline Features & $\begin{array}{l}\text { Total AIDs with } \\
\text { MS }(\boldsymbol{n}=111)\end{array}$ & $\begin{array}{l}\text { NLRP3 variants } \\
\text { with MS }(\boldsymbol{n}=19)\end{array}$ & $\begin{array}{l}\text { MEFV variants } \\
\text { with MS }(\boldsymbol{n}=34)\end{array}$ & $\begin{array}{l}\text { TNFRSF1A variants } \\
\text { with MS }(\boldsymbol{n}=58)\end{array}$ & $\begin{array}{l}\text { MS w/o mutations } \\
(\boldsymbol{n}=51)\end{array}$ \\
\hline M:F & $1: 2.2$ & $1: 1.7$ & $1: 2.4$ & $1: 3.4$ & $1: 2$ \\
Age at MS diagnosis (years) & $29.6 \pm 12.3$ & $31.5 \pm 11.5(11-48)$ & $28.4 \pm 12.3(7-63)$ & $28.9 .0 \pm 12.4(12-51)$ & $32.7 .0 \pm 11.7(14-56)$ \\
Diagnose latency for MS (years) & $3.3 \pm 5.8$ & $4.3 \pm 6.7(0-29)$ & $2.9 \pm 5.2(0-25)$ & $2.8 .0 \pm 4.0(0-29)$ & $2.3 .0 \pm 3.0(0-29)$ \\
MS disease duration (years) & $14.6 \pm 8.6$ & $12.2 \pm 8.6(3-35)$ & $13.8 \pm 8.5(3-27)$ & $17.7 \pm 8.8(5-32)$ & $14.0 \pm 7.8(8-43)$ \\
RRMS & $92 / 83 \%$ & $18 / 95 \%$ & $30 / 88 \%$ & $44 / 76 \%$ & $39 / 76 \%$ \\
SPMS & $12 / 11 \%$ & $1 / 5 \%$ & $2 / 6 \%$ & $9 / 16 \%$ & $7 / 14 \%$ \\
PPMS & $7 / 6 \%$ & $0 / 0 \%$ & $2 / 6 \%$ & $5 / 8 \%$ & $5 / 10 \%$ \\
EDSS at baseline & $3 \pm 2$ & $4 \pm 2$ & $3 \pm 2$ & $3 \pm 2$ & $3 \pm 2$ \\
EDSS at follow-up & $3 \pm 2$ & $3.5 \pm 2$ & $3.5 \pm 2$ & $3 \pm 2$ & $3.3 \pm 2$ \\
MSSS at baseline & $3.1 \pm 1.9$ & $4.4 \pm 2.6$ & $3.8 \pm 2.3$ & $3.8 \pm 2.6$ & $3.8 \pm 1.8$ \\
MSSS at follow-up & $3.1 \pm 1.9$ & $3.2 \pm 2.2$ & $3.4 \pm 1.7$ & $2.8 \pm 2$ & $2.9 \pm 1.5$ \\
DMT for mild disease course* & $67 / 60 \%$ & $10 / 53 \%$ & $28 / 82 \%$ & $29 / 50 \%$ & $35 / 68 \%$ \\
DMT for moderate/severe disease course** & $31 / 28 \%$ & $3 / 16 \%$ & $6 / 18 \%$ & $22 / 38 \%$ & $9 / 18 \%$ \\
No DMT & $13 / 12 \%$ & $6 / 32 \%$ & $0 / 0 \%$ & $7 / 12 \%$ & $12 / 21 \%$ \\
Positive family history for MS & $23 / 21 \%$ & $5 / 26 \%$ & $6 / 18 \%$ & $6 / 12 \%$
\end{tabular}

Demographic data of 108 MS patients were assessed. Numbers, if not stated differently, represent mean \pm SD (range). EDSS Expanded Disability Status Scale, MSSS Multiple Sclerosis Severity Score, RRMS relapsing-remitting MS, SPMS secondary progressive MS, PPMS primary progressive MS; DMT disease-modifying therapies *Interferons, glatirameracetat, teriflunomide, dimethylfumarate, steroids, and azathioprine

**Fingolimod, natalizumab, alemtuzumab, ocrelizumab, and rituximab 

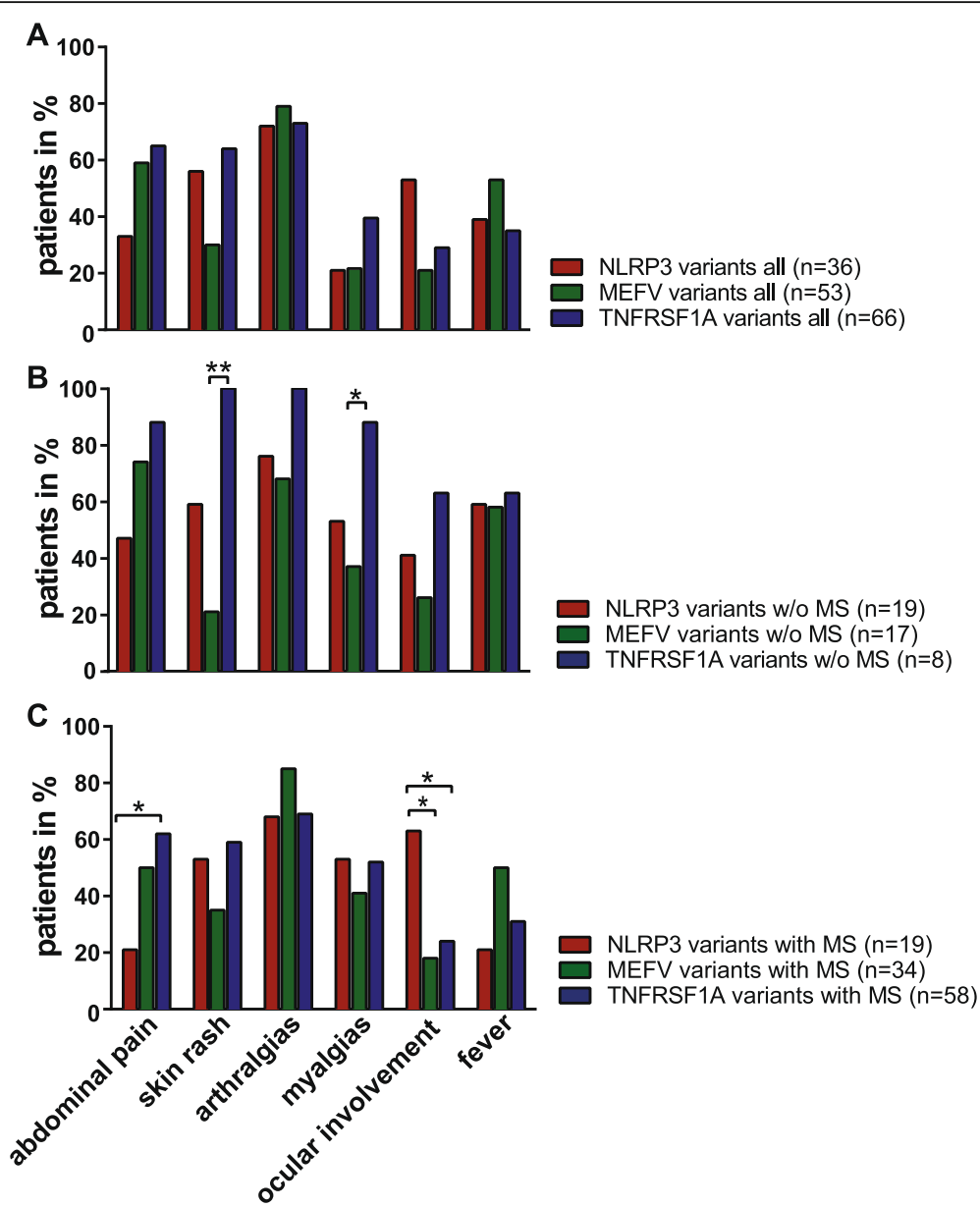

Fig. 6 Systemic symptoms. Systemic symptoms were distinguished as follows: abdominal pain, skin rash, arthralgias, myalgias, ocular involvement, fever in all patients (a) of NLRP3-, MEFV-, and TNFRSF1 low-penetrance variants w/o (b) or with MS (c). Numbers represent percentages

the low-penetrance Q703K variant compared to patients with other genotypes in our study is novel and unusual. In all our patients, a thorough and intensive diagnostic workup was performed and other potential differential diagnoses such as neurosarcoidosis or rheumatological diseases such as lupus erythematodes with CNS involvement were ruled out. However, we cannot exclude other genetic factors contributing to the severe phenotype as we neither performed whole-exome sequencing and nor excluded somatic mosaicism in our patients [41].

Looking in more detail on neurological manifestations in patients w/o MS, headache was the most prevalent symptom among low-penetrance variant carriers irrespective of the underlying genotype. This finding

Table 3 Laboratory data NLRP3-, MEFV-, and TNFRSF1A low-penetrance variants

\begin{tabular}{|c|c|c|c|c|c|c|c|}
\hline & All & $\begin{array}{l}\text { NLRP3 } \\
\text { variants } \\
\text { w/o MS }\end{array}$ & $\begin{array}{l}\text { NLRP3 } \\
\text { variants } \\
\text { with MS }\end{array}$ & $\begin{array}{l}\text { MEFV } \\
\text { variants } \\
\text { W/o MS }\end{array}$ & $\begin{array}{l}\text { MEFV } \\
\text { variants } \\
\text { with MS }\end{array}$ & $\begin{array}{l}\text { TNFRSF1A } \\
\text { variants } \\
\text { w/o MS }\end{array}$ & $\begin{array}{l}\text { TNFRSF1A } \\
\text { variants } \\
\text { with MS }\end{array}$ \\
\hline CRP (mg/dl) & $\begin{array}{l}2.7 \pm 1.6(54) \\
(0.6-86)\end{array}$ & $\begin{array}{l}2.2 \pm 2.0(7) \\
(0.6-10.1)\end{array}$ & $\begin{array}{l}2.1 \pm 1.2(3) \\
(0.8-2.8)\end{array}$ & $\begin{array}{l}4.7 \pm 8.9(10) \\
(0.65-86)\end{array}$ & $\begin{array}{l}1.7 \pm 1.5(16) \\
(0.6-11.5)\end{array}$ & $\begin{array}{l}0.7 \pm 0.1(2) \\
(0.7-0.8)\end{array}$ & $\begin{array}{l}4.7 \pm 6.6(16) \\
(0.6-23.8)\end{array}$ \\
\hline Leucocytes (G/I) & $13.3 \pm 0.6(36)(10.5-31.9)$ & $\begin{array}{l}13.5 \pm 2.4(6) \\
(10.3-29)\end{array}$ & $\begin{array}{l}14.1 \pm 3.1 \text { (4) } \\
(10.7-31.9)\end{array}$ & $\begin{array}{l}13.2 \pm 1.5(7) \\
(11.1-19.3)\end{array}$ & $\begin{array}{l}12.5 \pm 2.1(8) \\
(10.5-16.6)\end{array}$ & - & $\begin{array}{l}13.0 \pm 2.1(11) \\
(11.3-18.6)\end{array}$ \\
\hline SAA (mg/dl) & $\begin{array}{l}31 \pm 36.2(68) \\
(5.2-3115)\end{array}$ & $\begin{array}{l}172.2 \pm 492.9(11) \\
(5.2-3115)\end{array}$ & $\begin{array}{l}21.7 \pm 32.8(8) \\
(5.2-107)\end{array}$ & $\begin{array}{l}10.5 \pm 5.1 \\
(6.0-22.1)\end{array}$ & $\begin{array}{l}95 \pm 298.2(14) \\
(5.2-1130)\end{array}$ & $\begin{array}{l}9.4 \pm 3.0(5) \\
(5.7-13-1)\end{array}$ & $\begin{array}{l}18.4 \pm 25(21) \\
(5.2-122)\end{array}$ \\
\hline
\end{tabular}

Numbers represent mean \pm SD (sample size) and (range). CRP C-reactive protein (normal up to $0.5 \mathrm{mg} / \mathrm{dl}$ ); leucocytes (normal up to $10.4 \mathrm{G} / \mathrm{l}$ ); $\mathrm{SAA}$ serum amyloid-a (normal up to $5.0 \mathrm{mg} / \mathrm{l}$ ) 
demonstrates a similar result compared to AID patients carrying disease-causing variants, of whom headache syndromes were also reported at a high frequency such as $84 \%$ of CAPS and $72 \%$ of FMF patients [12, 39]. A greater proportion of patients with NLRP3 variants showed CN affection, which supports findings of our previous study and underscores the vulnerability of cranial nerves to inflammation caused by NLRP3-associated inflammasome activation [24]. Studies in animal models have shown that sensory neurons, including the trigeminal nucleus and ganglion, express NLRP3 which upon activation promotes IL-1 release and is blocked by MCC950, a specific NLRP3 inhibitor [42, 43]. Additional studies have also demonstrated that NLRP3 inflammasome activation within the cochlea and retina contributes to murine neuroinflammation [44, 45]. These observations may help explain the predominant affection of the vestibulocochlear nerve, the optic nerve, and the trigeminal nerves in patients with NLRP3 variants in our study.

The majority of patients in our cohort had concomitant MS. This and the fact that most patients were adults are readily explained by the fact that our outpatient clinic is specialized on the treatment of adult MS patients [13, 35]. Former studies already suggested that AID and MS are more likely to co-occur than expected by chance $[21,26$, 46]. Previous studies have identified the R92Q variant in the TNFRSF1A gene as a risk factor for MS [47]. The high prevalence of this variant in our study supports these observations. In contrast, although the Q703K NLRP3- and the E148Q MEFV variants have been described in MS patients $[24,28]$, they have not been confirmed as a susceptibility factor for MS so far. Interestingly, we observed both the K695R and the R408Q (in six cases as a compound heterozygous P369S-R408Q mutation) variants almost exclusively in MS patients, which warrants further investigation in larger study populations. Overall, the disease course of MS in AID patients was similar to MS patients without mutations. This suggests that the underlying genetic variants serve more as a susceptibility factor rather than being a distinct disease modifier in MS [24, 26, 28]. In addition, treatment with colchicine and anti-IL-1 therapy had no major influence on the disease course of MS in our small patient cohort.

It can be difficult to distinguish between "MS with coexisting AID" and "AID with CNS involvement" since the clinical presentation may be similar. Both disorders manifest with variable and episodic symptoms, often affect the optic nerve, and show white matter lesions on MRI as well as abnormalities in the CSF. Thus, careful evaluation and follow-up investigations are of great importance. CAPS, FMF, and TRAPS usually occur with multisystemic inflammation and therefore require medical care and treatment by a collaborative, multidisciplinary team. If at all, adult patients with a presumed diagnosis of an AID are seen by rheumatologists first. As a consequence, neurological manifestations may be overlooked, as neurological examinations as well as MRI and CSF analysis are not routinely performed in such settings.

Although we observed some differences concerning systemic symptoms, overlapping systemic manifestation makes it difficult for the clinician to distinguish between NLRP3-, MEFV-, or TNFRSF1A variants. Thus, if an AID is suspected, we propose to perform a multipaneled genetic diagnostic test as it is offered now in many laboratories. In addition, it can be challenging to differentiate AID from other rheumatological diseases. This often results in delayed diagnosis of AID, in fact, a majority of our patients had a mean diagnosis latency of 10 years.

The strength of this study is its sample size and its monocentric character, thereby circumventing the bias of multiple study sites. Obvious limitations, on the other hand, involve the retrospective character, recruitment bias (as an outpatient clinic specialized in MS), and the lack of patients with clear disease-causing mutations in the NLRP3- and TNFRSF1A gene for comparison. In addition, although the disease course of MS with and without genetic variants did not differ in our patient cohort, we cannot exclude an influence of these variants on MS development and progression. Since we did not collect the respective biomaterial from all our patients, we were unable to investigate and compare cytokine profiles and immune cell subtypes in sera and CSF in MS patients with and without NLRP3-, MEFV-, and TNFRSF1A low-penetrance variants. This is, however, of great interest since increased levels of IL-1ß have been reported in the CSF and in cerebral lesions of MS patients. Furthermore, IL-1 $\beta$ has recently been shown to correlate with cortical pathology load in MS at clinical onset $[48,49]$. Additionally, a role of the NLRP3 inflammasome has been demonstrated in the development of experimental autoimmune encephalomyelitis (EAE) [50]. Taken together, a more comprehensive immunophenotyping in correlation to clinical phenotypes in MS patients with and without AID is warranted and may elucidate the impact of these genetic variants on MS disease course in future studies.

\section{Conclusions}

Taken together, a broad spectrum of neurological manifestations including coexisting MS may occur in patients with low-penetrance variants of the TNFRSF1A-, NLRP3-, and $M E F V$ genes and both neurologists and rheumatologists should keep this in mind. Diagnosis of AID should be considered a potential differential diagnosis in patients with recurrent episodes of systemic inflammation with $\mathrm{CNS}$ involvement and unexplained $\mathrm{CN}$ affection. 


\section{Supplementary information}

Supplementary information accompanies this paper at https://doi.org/10. 1186/s12974-020-01867-5.

Additional file 1: Figure S1. Follow-up of AID patients w/o MS. Follow up time (in years) of NLRP3-, MEFV- and TNFRSF1A low penetrance variants w/o MS are depicted.

\section{Abbreviations}

AID: Autoinflammatory diseases; CAPS: Cryopyrin-associated periodic syndromes; CN: Cranial nerve affection; EDSS: Expanded Disability Status Scale; EAE: Autoimmune encephalomyelitis; FMF: Familial Mediterranean fever; IL-1ß: Interleukin-1 beta; MS: Multiple sclerosis; MSSS: Multiple Sclerosis Severity Scale; PPMS: Primary progressive MS; SPMS: Secondary progressive MS; TNF: Tumor necrosis factor; TRAPS: Tumor necrosis factor receptorassociated periodic syndrome; vs.: Versus; w/o: Without

\section{Acknowledgements}

We wish to thank H. Rodriguez and S. Mader for helpful comments on the manuscript and language editing; Sabine Pitter and Angelika Bamberger for patient care.

\section{Authors' contributions}

EM: acquisition, analysis, and interpretation of data; patient care and evaluation; and manuscript writing and editing. DW: acquisition, analysis, and interpretation of data and editing of the manuscript. JH, MS, IM, L.-A. G.: patient care and contribution to clinical data and editing of the manuscript. TK: development of study concept, study supervision, patient care and evaluation, analysis and interpretation of data, and writing, reviewing, and editing of the manuscript. All authors discussed the results and commented on the manuscript. The author(s) read and approved the final manuscript.

\section{Funding}

This work was supported by the Deutsche Forschungsgemeinschaft (German Research Foundation) within the framework of the Munich Cluster for Systems Neurology (EXC 1010 SyNergy) and the Clinical Competence Network for Multiple Sclerosis. JH is (partially) funded by the German Federal Ministry of Education and Research under 01ZZ1603[A-D] and 01ZZ1804[AH] (DIFUTURE).

\section{Availability of data and materials}

All data generated or analyzed during this study are included in this published article.

\section{Ethics approval and consent to participate}

The study was approved by the local ethics committee from the LudwigMaximilians University. All participants gave written informed consent (project 159-03, 163-16 and 600-15).

\section{Consent for publication}

Not applicable.

\section{Competing interests}

E. Mulazzani, D. Wagner, M. Schlüter, I. Meinl, and L.-A. Gerdes report no disclosures. J. Havla reports personal fees and non-financial support from Merck, Novartis, Roche, Santhera, Biogen, and Sanofi Genzyme and nonfinancial support of the Guthy-Jackson Charitable Foundation, all outside the submitted work

T. Kümpfel has received speaker honoraria including advisory boards from Bayer Healthcare, Teva Pharma, Merck, Novartis Pharma, Sanofi-Aventis/Genzyme, Roche Pharma, and Biogen as well as grant support from Novartis and Chugai Pharma in the past.

\section{Author details}

'Institute of Clinical Neuroimmunology, Biomedical Center and University Hospital, Ludwig-Maximilians University, Marchioninistraße 15, 81377 Munich, Germany. ${ }^{2}$ Munich Cluster for Systems Neurology (SyNergy), Munich, Germany.
Received: 19 December 2019 Accepted: 4 June 2020

Published online: 20 June 2020

\section{References}

1. Hoffman HM, Mueller $\mathrm{L}$, Broide DH, Wanderer AA, Kolodner RD. Mutation of a new gene encoding a putative pyrin-like protein causes familial cold autoinflammatory syndrome and Muckle-Wells syndrome. Nat Genet. 2001; 29(3):301-5.

2. Masters SL, Simon A, Aksentijevich I, Kastner DL. Horror autoinflammaticus: the molecular pathophysiology of autoinflammatory disease $\left(^{*}\right)$. Annu Rev Immunol. 2009:27:621-68.

3. Xu H, Yang J, Gao W, Li L, Li P, Zhang L, et al. Innate immune sensing of bacterial modifications of Rho GTPases by the Pyrin inflammasome. Nature. 2014;513(7517):237-41

4. de Torre-Minguela C, Mesa Del Castillo P, Pelegrin P. The NLRP3 and pyrin inflammasomes: implications in the pathophysiology of autoinflammatory diseases. Front Immunol. 2017:8:43.

5. Abbara S, Grateau G, Ducharme-Benard S, Saadoun D, Georgin-Lavialle S. Association of vasculitis and familial Mediterranean fever. Front Immunol. 2019:10:763.

6. Rebelo SL, Bainbridge SE, Amel-Kashipaz MR, Radford PM, Powell RJ, Todd I, et al. Modeling of tumor necrosis factor receptor superfamily $1 \mathrm{~A}$ mutants associated with tumor necrosis factor receptor-associated periodic syndrome indicates misfolding consistent with abnormal function. Arthritis Rheum. 2006;54(8):2674-87.

7. Probert L, Eugster HP, Akassoglou K, Bauer J, Frei K, Lassmann H, et al. TNFR1 signalling is critical for the development of demyelination and the limitation of T-cell responses during immune-mediated CNS disease. Brain. 2000;123(Pt 10):2005-19.

8. Caminero A, Comabella M, Montalban X. Tumor necrosis factor alpha (TNFalpha), anti-TNF-alpha and demyelination revisited: an ongoing story. J Neuroimmunol. 2011;234(1-2):1-6.

9. Stojanov S, Kastner DL. Familial autoinflammatory diseases: genetics, pathogenesis and treatment. Curr Opin Rheumatol. 2005;17(5):586-99.

10. Schwarzbach CJ, Schmitt WH, Szabo K, Bazner H, Hennerici MG, Blahak C. Chorea in a patient with cryopyrin-associated periodic syndrome. Neurology. 2016;86(3):241-4

11. Kitley JL, Lachmann HJ, Pinto A, Ginsberg L. Neurologic manifestations of the cryopyrin-associated periodic syndrome. Neurology. 2010;74(16):126770 .

12. Parker T, Keddie S, Kidd D, Lane T, Maviki M, Hawkins PN, et al. Neurology of the cryopyrin-associated periodic fever syndrome. Eur J Neurol. 2016;23(7): 1145-51.

13. Levy R, Gerard L, Kuemmerle-Deschner J, Lachmann HJ, Kone-Paut I, Cantarini $L$, et al. Phenotypic and genotypic characteristics of cryopyrinassociated periodic syndrome: a series of 136 patients from the Eurofever Registry. Ann Rheum Dis. 2015;74(11):2043-9.

14. Mamoudjy N, Maurey H, Marie I, Kone-Paut I, Deiva K. Neurological outcome of patients with cryopyrin-associated periodic syndrome (CAPS). Orphanet J Rare Dis. 2017:12(1):33.

15. Kilic H, Sahin S, Duman C, Adrovic A, Barut K, Turanli ET, et al. Spectrum of the neurologic manifestations in childhood-onset cryopyrin-associated periodic syndrome. Eur J Paediatr Neurol. 2019;23(3):466-72.

16. Schwabe AD, Monroe JB. Meningitis in familial Mediterranean fever. Am J Med. 1988:85(5):715-7.

17. Minden K, Aganna E, McDermott MF, Zink A. Tumour necrosis factor receptor associated periodic syndrome (TRAPS) with central nervous system involvement. Ann Rheum Dis. 2004;63(10):1356-7.

18. Wildemann B, Rudofsky G Jr, Kress B, Jarius S, Konig F, Schwenger V. The tumor-necrosis-factor-associated periodic syndrome, the brain, and tumornecrosis-factor-alpha antagonists. Neurology. 2007;68(20):1742-4.

19. Compeyrot-Lacassagne S, Tran TA, Guillaume-Czitrom S, Marie I, Kone-Paut I. Brain multiple sclerosis-like lesions in a patient with Muckle-Wells syndrome Rheumatology (Oxford). 2009;48(12):1618-9.

20. Topcuoglu MA, Karabudak R. Familial Mediterranean fever and multiple sclerosis. J Neurol. 1997:244(8):510-4.

21. Akman-Demir G, Gul A, Gurol E, Ozdogan H, Bahar S, Oge AE, et al. Inflammatory/ demyelinating central nervous system involvement in familial Mediterranean fever (FMF): coincidence or association? J Neurol. 2006;253(7):928-34.

22. Lidar M, Brantz Y, Shinar Y, Reznik-Wolf H, Livneh A, Ben Zvi I, et al. A high and equal prevalence of the Q703K variant in NLRP3 patients with 
autoinflammatory symptoms and ethnically matched controls. Clin Exp Rheumatol. 2017;35(Suppl 108(6)):82-5.

23. Gattorno M, Hofer M, Federici S, Vanoni F, Bovis F, Aksentijevich I, et al. Classification criteria for autoinflammatory recurrent fevers. Ann Rheum Dis. 2019;78(8):1025-32.

24. Schuh E, Lohse P, Ertl-Wagner B, Witt M, Krumbholz M, Frankenberger M, et al. Expanding spectrum of neurologic manifestations in patients with NLRP3 low-penetrance mutations. J Neuroinflammation. 2015;2(4):e109.

25. Havla J, Lohse P, Gerdes LA, Hohlfeld R, Kumpfel T. Symptoms related to tumo necrosis factor receptor 1-associated periodic syndrome, multiple sclerosis, and severe rheumatoid arthritis in patients carrying the TNF receptor superfamily 1A D12E/p.Asp41Glu mutation. J Rheumatol. 2013;40(3):261-4.

26. Kumpfel T, Hoffmann LA, Rubsamen H, Pollmann W, Feneberg W, Hohlfeld $\mathrm{R}$, et al. Late-onset tumor necrosis factor receptor-associated periodic syndrome in multiple sclerosis patients carrying the TNFRSF1A R92Q mutation. Arthritis Rheum. 2007;56(8):2774-83.

27. Kumpfel T, Hoffmann LA, Pellkofer H, Pollmann W, Feneberg W, Hohlfeld R, et al. Multiple sclerosis and the TNFRSF1A R92Q mutation: clinical characteristics of 21 cases. Neurology. 2008;71(22):1812-20.

28. Kumpfel T, Gerdes LA, Wacker T, Blaschek A, Havla J, Krumbholz M, et al. Familial Mediterranean fever-associated mutation pyrin E148Q as a potential risk factor for multiple sclerosis. Mult Scler. 2012;18(9):1229-38.

29. Hohne C, Schuh E, Kumpfel T, Straube A. Cryopyrin-associated periodic fever syndrome manifesting as Tolosa-Hunt syndrome. Cephalalgia. 2016;36(14): 1392-6.

30. Roxburgh RH, Seaman SR, Masterman T, Hensiek AE, Sawcer SJ, Vukusic S, et al. Multiple Sclerosis Severity Score: using disability and disease duration to rate disease severity. Neurology. 2005;64(7):1144-51.

31. Aksentijevich I, Putnam CD, Remmers EF, Mueller JL, Le J, Kolodner RD, et al. The clinical continuum of cryopyrinopathies: novel CIAS1 mutations in North American patients and a new cryopyrin model. Arthritis Rheum. 2007;56(4):1273-85.

32. Verma $D$, Lerm $M$, Blomgran Julinder $R$, Eriksson $P$, Soderkvist $P$, Sarndahl $E$. Gene polymorphisms in the NALP3 inflammasome are associated with interleukin-1 production and severe inflammation: relation to common inflammatory diseases? Arthritis Rheum. 2008;58(3):888-94.

33. Naselli A, Penco F, Cantarini L, Insalaco A, Alessio M, Tommasini A, et al. Clinica characteristics of patients carrying the Q703K variant of the NLRP3 gene: a 10year multicentric national study. J Rheumatol. 2016;43(6):1093-100.

34. Vitale A, Lucherini OM, Galeazzi M, Frediani B, Cantarini L. Long-term clinical course of patients carrying the Q703K mutation in the NLRP3 gene: a case series. Clin Exp Rheumatol. 2012;30(6):943-6.

35. Lachmann HJ, Papa R, Gerhold K, Obici L, Touitou I, Cantarini L, et al. The phenotype of TNF receptor-associated autoinflammatory syndrome (TRAPS) at presentation: a series of 158 cases from the Eurofever/EUROTRAPS international registry. Ann Rheum Dis. 2014;73(12):2160-7.

36. Ben-Chetrit E, Lerer I, Malamud E, Domingo C, Abeliovich D. The E148Q mutation in the MEFV gene: is it a disease-causing mutation or a sequence variant? Hum Mutat. 2000;15(4):385-6.

37. Schuh E, Gross CJ, Wagner D, Schluter M, Gross O, Kumpfel T. MCC950 blocks enhanced interleukin-1 beta production in patients with NLRP3 low penetrance variants. Clin Immunol. 2019;203:45-52.

38. Verma D, Sarndahl E, Andersson H, Eriksson P, Fredrikson M, Jonsson Jl, et al. The Q705K polymorphism in NLRP3 is a gain-of-function alteration leading to excessive interleukin-1beta and IL-18 production. PLoS One. 2012;7(4):e34977.

39. Canpolat M, Gumus H, Gunduz Z, Dusunsel R, Kumandas S, Bayram AK, et al. Neurological manifestations in familial Mediterranean fever: results of 22 children from a reference center in Kayseri, an urban area in Central Anatolia, Turkey. Neuropediatrics. 2017;48(2):79-85.

40. Gedalia A, Zamir S. Neurologic manifestations in familial Mediterranean fever. Pediatr Neurol. 1993;9(4):301-2.

41. Nishikomori R, Izawa K, Kambe N, Ohara O, Yasumi T. Low-frequency mosaicism in cryopyrin-associated periodic fever syndrome: mosaicism in systemic autoinflammatory diseases. Int Immunol. 2019;31(10):649-55.

42. Santoni G, Cardinali C, Morelli MB, Santoni M, Nabissi M, Amantini C. Danger- and pathogen-associated molecular patterns recognition by pattern-recognition receptors and ion channels of the transient receptor potential family triggers the inflammasome activation in immune cells and sensory neurons. J Neuroinflammation. 2015;12:21.

43. He W, Long T, Pan Q, Zhang S, Zhang Y, Zhang D, et al. Microglial NLRP3 inflammasome activation mediates $\mathrm{\|}$-1 1 beta release and contributes to central sensitization in a recurrent nitroglycerin-induced migraine model. J Neuroinflammation. 2019;16(1):78.

44. Puyang Z, Feng L, Chen H, Liang P, Troy JB, Liu X. Retinal ganglion cell loss is delayed following optic nerve crush in NLRP3 knockout mice. Sci Rep. 2016;6:20998.

45. Nakanishi H, Kawashima Y, Kurima K, Chae JJ, Ross AM, Pinto-Patarroyo G, et al. NLRP3 mutation and cochlear autoinflammation cause syndromic and nonsyndromic hearing loss DFNA34 responsive to anakinra therapy. Proc Natl Acad Sci U S A. 2017;114(37):E7766-E75.

46. Blaschek A, Kries RV, Lohse P, Huss K, Vill K, Belohradsky BH, et al. TNFRSF1A and MEFV mutations in childhood onset multiple sclerosis. Eur J Paediatr Neurol. 2018;22(1):72-81

47. De Jager PL, Jia X, Wang J, de Bakker PI, Ottoboni L, Aggarwal NT, et al. Metaanalysis of genome scans and replication identify CD6, IRF8 and TNFRSF1A as new multiple sclerosis susceptibility loci. Nat Genet. 2009;41(7):776-82.

48. Seppi D, Puthenparampil M, Federle L, Ruggero S, Toffanin E, Rinaldi F, et al. Cerebrospinal fluid IL-1 beta correlates with cortical pathology load in multiple sclerosis at clinical onset. J Neuroimmunol. 2014;270(1-2):56-60.

49. Hauser SL, Doolittle TH, Lincoln R, Brown RH, Dinarello CA. Cytokine accumulations in CSF of multiple sclerosis patients: frequent detection of interleukin-1 and tumor necrosis factor but not interleukin-6. Neurology. 1990:40(11):1735-9.

50. Inoue M, Williams KL, Gunn MD, Shinohara ML. NLRP3 inflammasome induces chemotactic immune cell migration to the CNS in experimental autoimmune encephalomyelitis. Proc Natl Acad Sci U S A. 2012;109(26):10480-5.

\section{Publisher's Note}

Springer Nature remains neutral with regard to jurisdictional claims in published maps and institutional affiliations.

\section{Ready to submit your research? Choose BMC and benefit from:}

- fast, convenient online submission

- thorough peer review by experienced researchers in your field

- rapid publication on acceptance

- support for research data, including large and complex data types

- gold Open Access which fosters wider collaboration and increased citations

- maximum visibility for your research: over $100 \mathrm{M}$ website views per year

At $\mathrm{BMC}$, research is always in progress.

Learn more biomedcentral.com/submission 